ELECTRONIC RESEARCH ANNOUNCEMENTS

OF THE AMERICAN MATHEMATICAL SOCIETY

Volume 1, Issue 2, 1995

\title{
ON QUANTUM LIMITS ON FLAT TORI
}

\author{
DMITRY JAKOBSON
}

(Communicated by Yitzhak Katznelson)

\begin{abstract}
We classify all weak $*$ limits of squares of normalized eigenfunctions of the Laplacian on two-dimensional flat tori (we call these limits quantum limits). We also obtain several results about such limits in dimensions three and higher. Many of the results are a consequence of a geometric lemma which describes a property of simplices of codimension one in $\mathbb{R}^{n}$ whose vertices are lattice points on spheres. The lemma follows from the finiteness of the number of solutions of a system of two Pell equations. A consequence of the lemma is a generalization of the result of $\mathrm{B}$. Connes. We also indicate a proof (communicated to us by J. Bourgain) of the absolute continuity of the quantum limits on a flat torus in any dimension. We generalize a two-dimensional result of Zygmund to three dimensions; we discuss various possible generalizations of that result to higher dimensions and the relation to $L^{p}$ norms of the densities of quantum limits and their Fourier series.
\end{abstract}

In this note we shall consider eigenfunctions of the Laplacian on the standard flat $d$-dimensional torus $\mathbb{T}^{d}=\mathbb{R}^{d} / \mathbb{Z}^{d}$. Most of the results of this paper are also valid for other flat tori $\mathbb{R}^{d} / \Lambda$, where $\Lambda$ is a lattice (of rank $d$ ) in $\mathbb{R}^{d}$ different from $\mathbb{Z}^{d}$.

Let $\varphi$ be an eigenfunction with the eigenvalue $\lambda$, and let $\|\varphi\|_{2}=1$. The multiplicity of $\lambda$ is equal to the number of lattice points on a sphere of radius $\sqrt{\lambda}$ centered at the origin (or, equivalently, to the number of ways of writing $\lambda$ as a sum of $d$ squares). It is well-known that the multiplicity is unbounded for $d \geq 2$.

We are interested in all possible weak limits as $\lambda_{j} \rightarrow \infty$ of the (probability) measures $d \mu_{j}=\left|\varphi_{j}\right|^{2} d \mathrm{vol}$ (where $d \mathrm{vol}$ is the Riemannian volume form on $\mathbb{T}^{d}$ ), which are henceforth called quantum limits (cf. [Jak]).

A. Zygmund proved in $[\mathrm{Zyg}]$ that for $d=2$

$$
\|\varphi\|_{4} /\|\varphi\|_{2} \leq 5^{1 / 4}
$$

It follows that for $d=2$ any quantum limit $d \nu$ is absolutely continuous, and its density is a function $\psi \in L^{2}$ satisfying $\|\psi\|_{2} \leq 5^{1 / 2}$. In fact, much more can be said about the densities of quantum limits on two-dimensional tori.

1991 Mathematics Subject Classification: 42B05, 81Q50, 58C40, 52B20, 11D09, 11J86

Key words and phrases: flat tori, Laplacian, resonances, Pell equation, weak $*$ limits, Fourier series

Received by the editors April 20, 1995, and, in revised form, July 19, 1995.

(C)1995 American Mathematical Society 
To formulate our result, denote the Fourier expansion of a quantum limit $d \nu$ on $\mathbb{T}^{d}$ by

$$
d \nu \sim \sum_{\tau \in \mathbb{Z}^{d}} c_{\tau} e^{i(\tau, x)},
$$

where $(\tau, x)$ is the usual scalar product in $\mathbb{R}^{d}$.

We prove the following result:

Theorem 1. The density of every quantum limit $d \nu$ on $\mathbb{T}^{2}$ is a trigonometric polynomial all of whose non-zero frequencies lie on the union of (at most) two circles centered at the origin.

In other words, for every $d \nu$ with the Fourier expansion (2) there exist two positive numbers $r_{1}$ and $r_{2}$ such if $\tau \neq \mathbf{0}$ and $c_{\tau} \neq 0$ then either $|\tau|=r_{1}$ or $|\tau|=r_{2}$. We can also describe the combinations of $\tau$-s (as above) that occur (together with the set of possible $c_{\tau}$-s).

The first question one asks for $d>2$ is whether quantum limits are absolutely continuous with respect to Lebesgue measure or not. No analogue of (1) is known for $d>2$, so the absolute continuity of quantum limits has to be proved differently than for $d=2$. J. Bourgain has communicated to us the proof of

Theorem 2 (Bourgain). Quantum limits are absolutely continuous in any dimension.

The proof involves repeated applications of a result due to B. Connes ${ }^{1}$ (see Proposition 3 below); a property ${ }^{2}$ of sequences of nonnegative numbers uniformly bounded in $l^{1}$ is also used.

The rest of this note is concerned with obtaining more precise information about the possible quantum limits in dimensions three and higher, although we are not able to classify all such limits as in dimension two.

The first result is

Theorem 3. For $3 \leq d \leq 5$, every quantum limit on $\mathbb{T}^{d}$ satisfies

$$
\sum_{\tau \in \mathbb{Z}^{d}}\left|c_{\tau}\right|^{d-2}<\infty
$$

(where $c_{\tau}$-s are defined by (2)).

We conjecture that (3) holds in any dimension $d>5$, but are not able to prove it because of certain technical difficulties.

It follows from Theorem 3 that the density of any quantum limit on $\mathbb{T}^{3}$ is a function that has absolutely convergent Fourier series; for $d=4$ it follows that any such function is in $L^{2}$. We also deduce from a certain conjecture about the distribution of $n$-tuples of primes $^{3}$ (which we shall call conjecture $H L$, cf. [Jak]) that on a three-dimensional torus there exist quantum limits which are not trigonometric polynomials; on a four-dimensional torus we can prove this unconditionally.

\footnotetext{
${ }^{1} \mathrm{~J}$. Bourgain has remarked that Proposition 3 also follows from lemma 25 in [Bou2].

${ }^{2}$ Sometimes referred to as a "sequence splitting lemma".

${ }^{3} \mathrm{~A}$ weak form of Hardy-Littlewood's conjecture, cf. [HL].
} 
By Theorem 2, every quantum limit has a density which is a (nonnegative) function in $L^{1}\left(\mathbb{T}^{d}\right)$. The question arises whether the densities of quantum limits on $\mathbb{T}^{d}$ belong to $L^{p}$ for any $p>1$ and $d \geq 5$. The answer turns out to be intimately related to the generalization of Zygmund's result (1) to higher dimensions.

Namely, we define the number $A(d, p) \leq \infty$ by

$$
A(d, p)=\sup _{\Delta \varphi+\lambda \varphi=0}\left(\|\varphi\|_{p} /\|\varphi\|_{2}\right)
$$

(where the supremum is taken over the eigenfunctions of the Laplacian on $\mathbb{T}^{d}$ ). ${ }^{4}$ It is not known whether $A(d, p)<\infty$ for any $p>2$ and $d \geq 3$. Bourgain showed in [Bou1] that $A(d, p)=\infty$ for $d \geq 4$ and $p \geq 2(d+1) /(d-3)$.

We can show that if $A(d, p)=\infty$ for some $p>2$, then there exist quantum limits on $\mathbb{T}^{d+3}$ whose densities are not in $L^{p / 2}\left(\mathbb{T}^{d+3}\right)$.

To state the converse result, for $d \geq 2$ we define $B(d, p)$ by

$$
B(d, p)=\sup \left\{\|\varphi\|_{p} /\|\varphi\|_{2}: \widehat{\varphi} \in\left(\Omega \cap \mathbb{Z}^{d+2}\right)\right\}
$$

where the supremum is taken over the trigonometric polynomials on $\mathbb{T}^{d+2}$ all of whose frequencies lie on an intersection of $\mathbb{Z}^{d+2}$ with a $(d-1)$-dimensional sphere $\Omega$ lying in a $d$-dimensional subspace of $\mathbb{R}^{d+2}$, and the $L^{p}$ norms are for the functions on $\mathbb{T}^{d+2}$. It is easy to see ${ }^{5}$ that $B(d, p) \geq A(d, p)$. One can generalize (1) and prove that $B(2,4)<\infty$. It may happen that $B(d, p)<\infty$ whenever $A(d, p)<\infty$, but we cannot prove this.

We can show that if $B(d, p)<\infty$ for some $d$ and $p>2$, then the density of any quantum limit on $\mathbb{T}^{d+2}$ is in $L^{p / 2}\left(\mathbb{T}^{d+2}\right)$.

Let $\varphi$ be the eigenfunction of the Laplacian with the eigenvalue $\lambda$. We want to prove an analogue of (1) for the Fourier series of the function $|\varphi|^{2}$. The Fourier expansion of $\varphi$ is given by

$$
\varphi(x)=\sum_{\xi \in \mathbb{Z}^{d} ;|\xi|^{2}=\lambda} a_{\xi} e^{i(\xi, x)}, a_{\xi} \in \mathbb{C}
$$

It follows from the previous formula that

$$
|\varphi|^{2}=\sum_{\tau} b_{\tau} e^{i(\tau, x)}, \quad b_{\tau}=\sum_{\substack{\xi-\eta=\tau \\|\xi|^{2}=|\eta|^{2}=\lambda}} a_{\xi} \bar{a}_{\eta}
$$

If $A(d, p)<\infty$ for some $2<p=p(d) \leq 4$, then by Hausdorff-Young theorem

$$
\left(\sum_{\tau \in \mathbb{Z}^{d}}\left|b_{\tau}\right|^{q}\right)^{1 / q} \ll\|\varphi\|_{2}^{2}
$$

uniformly on $\mathbb{T}^{d}$ for $q=q(d)=p(d) /(p(d)-2)$.

\footnotetext{
${ }^{4}$ The question of the rate of growth of the ratio in (4) with increasing eigenvalue is one of the basic questions in quantum chaos, cf. [Sar].

${ }^{5}$ We can identify the eigenfunctions on $\mathbb{T}^{d}$ with the eigenfunctions on $\mathbb{T}^{d+2}$ all of whose frequencies lie in the subspace $\left\{x \in \mathbb{R}^{d+2} \mid x_{d+1}=x_{d+2}=0\right\}$ of $\mathbb{R}^{d+2}$.
} 
Accordingly, we define a number $C(d, q) \leq \infty$ by

$$
C(d, q)=\sup _{\Delta \varphi+\lambda \varphi=0}\left(\|\widehat{f}\|_{q} /\|\varphi\|_{2}^{2}\right), \quad f=|\varphi|^{2}
$$

where $\widehat{f}$ is the Fourier series of $f$, and the supremum is taken over the eigenfunctions of the Laplacian on $\mathbb{T}^{d}$.

We have just shown that for $2 \leq p \leq 4,(A(d, p))^{2} \geq C(d, p /(p-2))$. Zygmund proves in [Zyg] that $C(2,2)<\infty$. Note that Bourgain's results in [Bou1] suggest that if $C(d, q(d))<\infty$, then $q(d) \rightarrow \infty$ as $d \rightarrow \infty$, and this can indeed be shown.

We prove

Proposition 1. On a three-dimensional torus, $C(3,3)<\infty$.

The proof is by a geometric argument in the spirit of $[\mathrm{Zyg}]$. However, we cannot use Proposition 1 to bound $\|\varphi\|_{p}$ uniformly for any $p>2$ because the exponent $q$ in our bound is bigger than two. The ideas of the proof of Proposition 1 should allow to prove that $C(d, d)<\infty$; however, for $d \geq 3$ certain technical difficulties arise which at the moment prevent us from extending Proposition 1 to dimensions four and higher.

We can show that if $C(d, q)=\infty$, then there exist such quantum limits on $\mathbb{T}^{d+3}$ that the Fourier series of their densities are not in $l^{q}$.

To state the converse result, for $d \geq 2$ we define $D(d, q)$ by

$$
D(d, q)=\sup \left\{\|\widehat{f}\|_{q} /\|\varphi\|_{2}^{2}: \widehat{\varphi} \in\left(\Omega \cap \mathbb{Z}^{d+2}\right)\right\}, \quad f=|\varphi|^{2}
$$

where the supremum is taken over the same trigonometric polynomials on $\mathbb{T}^{d+2}$ as in the definition of $B(d, p)$. It is easy to see that $D(d, q) \geq C(d, q)$ and that for $2 \leq p \leq 4,(B(d, p))^{2} \geq D(d, p /(p-2))$.

We can show that if $D(d, q)<\infty$, then for any quantum limit $d \nu$ on $\mathbb{T}^{d+2}$ given by $(2)$

$$
\sum_{\tau \in \mathbb{Z}^{d+2}}\left|c_{\tau}\right|^{q}<\infty
$$

One can also generalize the proofs of (1) and Proposition 1 and show that $D(2,2)<$ $\infty$ and $D(3,3)<\infty$, thus proving Theorem 1 for $d=4$ and $d=5$.

Finally, we discuss the rate of growth on $\mathbb{T}^{d}$ of the sum

$$
\Sigma(\rho)=\sum_{\tau \in \mathbb{Z}^{d} ;|\tau|<\rho}\left|c_{\tau}\right|
$$

Conjecture HL implies that on the four-dimensional torus there exist quantum limits for which $\Sigma(\rho) \rightarrow \infty$ as $\rho \rightarrow \infty$; we can prove this unconditionally for the five-dimensional torus. We prove

Proposition 2. On $\mathbb{T}^{d}, \Sigma(\rho) \ll \rho^{d-3}$ for $d \geq 6$. For $d=5, \Sigma(\rho) \ll \rho^{3 / 2+\varepsilon}$ and for $d=4, \Sigma(\rho) \ll \rho^{\varepsilon}$ for every $\varepsilon>0$.

We expect that $\Sigma(\rho) \ll \rho^{d-4+\varepsilon}$ for any $\varepsilon>0$. Conjecture HL implies that for $d \geq 5$ and for any $\varepsilon>0$,

$$
\varlimsup_{\rho \rightarrow \infty} \frac{\Sigma(\rho)}{\rho^{d-4-\varepsilon}}=\infty
$$


we can also prove unconditionally that for $d \geq 6, \varlimsup_{\rho \rightarrow \infty} \Sigma(\rho) / \rho^{d-5-\varepsilon}=\infty$.

Many of the above facts are consequences of a Geometric lemma which is of independent interest. To motivate the lemma, note that it follows from (5) that if $\varphi$ is the eigenfunction of the Laplacian on $\mathbb{T}^{d}$ with the eigenvalue $\lambda$, then the nonzero frequencies of $|\varphi|^{2}$ are given by the chords connecting the lattice points on the sphere $S$ of radius $\sqrt{\lambda}$ in $\mathbb{R}^{d}$ (centered at the origin). Also, the size of the Fourier coefficient $b_{\tau}$ is determined by the coefficients $a_{\xi}, a_{\eta}$ corresponding to such pairs $(\xi, \eta)$ of lattice points on $S$ that the chord $\xi-\eta$ connecting them is equal to $\tau$. We will call such pairs $\tau$-resonances (the terminology comes from mechanics, where it is usually assumed that $|\tau| \ll \sqrt{\lambda}$ ).

Given a measure $d \nu$ on $\mathbb{T}^{d}$ with the Fourier expansion (2), and a sequence $\varphi_{j}$ of eigenfunctions of the Laplacian with (increasing) eigenvalues $\lambda_{j}$, by $\left|\varphi_{j}\right|^{2} \rightarrow d \nu$ weak $*$ as $j \rightarrow \infty$ we shall mean that for every $\tau, b_{\tau}(j) \rightarrow c_{\tau}$ as $j \rightarrow \infty$ (for $\tau=\mathbf{0}$, $b_{\tau}(j)$ is always equal to 1 , so $\left.c_{\mathbf{0}}=1\right)$. Thus we are interested in $\tau$-resonances which appear on every sphere of radius $\sqrt{\lambda_{j}}$ for $j>N=N(\tau)$.

To state the Geometric lemma, we give a definition. Let $\Delta$ be a $d$-dimensional simplex with $d+1$ vertices all lying in $\mathbb{Z}^{n}$ for some $n$ (i.e. a line segment for $d=1$, a triangle for $d=2$ etc).

Definition. The points $\xi_{1}, \xi_{2}, \ldots, \xi_{d+1} \in \mathbb{Z}^{n}$ lying on a sphere in $\mathbb{R}^{n}$ are called $\Delta$-resonant if they form a d-dimensional simplex which can be translated into $\Delta$.

This definition is just a multi-dimensional generalization of $\tau$-resonances defined above for $d=1$. We now state the lemma.

Geometric lemma. Let $\Delta_{1}, \ldots, \Delta_{k}$ be a collection of d-dimensional simplices with vertices in $\mathbb{Z}^{d+1}$, and let $S_{1}, \ldots, S_{n}, \ldots$ be an infinite sequence of d-dimensional spheres in $\mathbb{R}^{d+1}$ centered at the origin with increasing radii, such that on each sphere $S_{n}$ there are $\Delta_{j}$-resonant points for every $1 \leq j \leq k$. Then the radii of the circumscribed spheres $\Omega_{j}$ of $\Delta_{j}$-s can take (at most) two different values.

For $d=1$ this just means that the line segments $\Delta_{1}, \ldots, \Delta_{k}$ can have at most two different lengths (hence at most two different circles in Theorem 1). The lemma is proved by first reducing the statement to counting the number of solutions of a system of Pell equations, and then using the (known) fact that this number is finite when the coefficients of the equations are quadratically independent. This fact can be proved either by applying Baker's results on linear froms in logarithms (cf. [Bak1] and [Bak2]; also, cf. [Mi] for the proof of a similar statement); or by applying Siegel's theorem about the finiteness of number of integer points on elliptic curves. The second proof, though easier, does not produce effective bounds obtained from the first proof.

The Geometric lemma proves a certain finiteness statement about the simplices of codimension one in $\mathbb{R}^{n}$ whose vertices are lattice points on spheres. One can ask whether it is possible to prove an analogous finiteness statements for simplices of codimension two or more. The answer to the above question is negative. Namely, conjecture HL implies that there exists an infinite sequence $\Delta_{j}$ of simplices with vertices in $\mathbb{Z}^{n}$ of codimension two, and a sequence $S_{k}$ of spheres in $\mathbb{R}^{n}$ centered at the origin of increasing radii such that on $S_{k}$ there are $\Delta_{j}$-resonant points for $1 \leq j \leq k$ (this implies the existence of quantum limits on $\mathbb{T}^{3}$ which are not trigonometric polynomials); an analogous statement for simplices of codimension three can be proved unconditionally. 
We can prove analogues of the Geometric lemma for lattices in $S L(n, \mathbb{R})$; these results will be discussed elsewhere.

The main application of the Geometric lemma is Proposition 4 given below. It is a generalization of a result of B. Connes (cf. [Con]). To state the result, we first define certain graphs on spheres.

Definition. For a d-dimensional sphere $S$ in $\mathbb{R}^{n}$ and for $\rho>0$, define $G_{\rho}(S)$ to be the graph with vertices at the points of $\mathbb{Z}^{n}$ located on $S$ obtained by connecting two vertices whenever the distance between them is less than $\rho$. Also, for $\rho>\rho_{1}>0$ define $G_{\rho}^{\rho_{1}}(S)$ to be the graph with the same vertices, and with two vertices $\xi$ and $\eta$ connected whenever $\rho_{1}<|\xi-\eta|<\rho$.

We remark that $G_{\rho}^{\rho_{1}}(S)$ coincides with $G_{\rho}(S)$ for $\rho_{1}=0$.

We now state Connes' result:

Proposition 3 (Connes). There exists a (positive) function $g=g_{d}(R) \asymp R^{2 /(d+2) \text { ! }}$ such that on any $d$-dimensional sphere $S$ in $\mathbb{R}^{n}$ of radius $R$, for any $0<\rho<g_{d}(R)$ (or, equivalently, $R \gg \rho^{(d+2) ! / 2}$ ) the connected components of $G_{\rho}(S)$ all lie on one of the $(d-1)$-dimensional spheres $\Omega_{1}, \Omega_{2}, \ldots, \Omega_{m}$ formed by the intersection of $S$ with d-dimensional planes in $\mathbb{R}^{n}$.

Using Proposition 3 and the Geometric lemma, we prove

Proposition 4. Given a sequence of spheres $S_{j}$ (of radius $R_{j}$ ) in $\mathbb{R}^{d+1}$ centered at the origin, there exist a subsequence $\left\{j_{k}\right\}$ of $\{j\}$, and a number $\rho_{1} \geq 0$ with the following property: given $\rho>\rho_{1}$, there exists $N \in \mathbb{N}$ such that if we take any sphere $S=S_{j_{k}}$ with $j_{k}>N$ and consider the graph $G=G_{\rho}^{\rho_{1}}(S)$, then the connected components of $G$ will lie on one of $(d-2)$-dimensional spheres $\Omega_{1}, \Omega_{2}, \ldots, \Omega_{m}, m=$ $m\left(j_{k}\right)$ (formed by the intersections of $S$ with the subspaces of $\mathbb{R}^{d+1}$ of codimension two).

Proposition 4 thus allows us to "reduce the dimension by two" when proving statements about the quantum limits.

The proofs of Theorem 1, Geometric lemma and Proposition 4, and of all the results about the quantum limits in dimensions three and four (except for Proposition 1) appear in [Jak]. Further details of this work will be published elsewhere.

Acknowledgements. This problem arose in conversations with Professor Shnirelman and Professor Sarnak at Princeton in the Fall of 1994. It is a pleasure for the author to thank Professors Sarnak, Rudnick, Cordoba and Shnirelman for helpful discussions and encouragements during this research, and Professor Bourgain for the permission to present Theorem 2 .

\section{BIBLIOGRAPHY}

[Bak1] A. Baker, Transcedental number theory, Cambridge University Press, 1975.

[Bak2] A. Baker, A sharpening of the bounds for linear forms in logarithms II., Acta Arithmetica XXIV (1973), 33-36.

[Bou1] J. Bourgain, Eigenfunction bounds for the Laplacian on the n-torus., Intern. Math. Res. Notices, No.3 (1993), 61-66.

[Bou2] J. Bourgain, Quasi-periodic solutions of Hamiltonian perturbations of 2D linear Shroödinger equations, preprint (1994).

[Con] B. Connes, Sur les coefficients des séries trigonométriques convergents sphériquement, C. R. Acad. Sc. Paris, 283 (1976), A159-A161. 
[HL] G. Hardy and J. Littlewood, Some problems of 'partitio numeronum'; III: on the expression of a number as a sum of primes, Collected Papers of G. H. Hardy, Oxford University Press, 1966, pp. 561-630.

[Jak] D. Jakobson, Ph. D. Thesis, Princeton University (1995).

[Mi] M. Mignotte, Intersection des images de certaines suites récurrentes linéaires, Theor. Computer Science, No. 7 (1978), 117-122.

[Sar] P. Sarnak, Arithmetic Quantum Chaos, Blyth Lectures, Toronto (1993).

[Zyg] A. Zygmund, On Fourier coefficients and Transforms of functions of two variables, Studia Mathematica, T.L. (1974), 189-201.

Department of Mathematics, Princeton University, Princeton, NJ 08544

E-mail address:diy@math.princeton.edu 